This item was submitted to Loughborough's Research Repository by the author.

Items in Figshare are protected by copyright, with all rights reserved, unless otherwise indicated.

\title{
A mixed-cost blind adaptive receiver for DS-CDMA
}

PLEASE CITE THE PUBLISHED VERSION

PUBLISHER

(C) IEEE

VERSION

VoR (Version of Record)

LICENCE

CC BY-NC-ND 4.0

REPOSITORY RECORD

Yuvapoositanon, Peerapol, and Jonathon Chambers. 2019. "A Mixed-cost Blind Adaptive Receiver for DSCDMA". figshare. https://hdl.handle.net/2134/5793. 
This item was submitted to Loughborough's Institutional Repository (https://dspace.lboro.ac.uk/) by the author and is made available under the following Creative Commons Licence conditions.

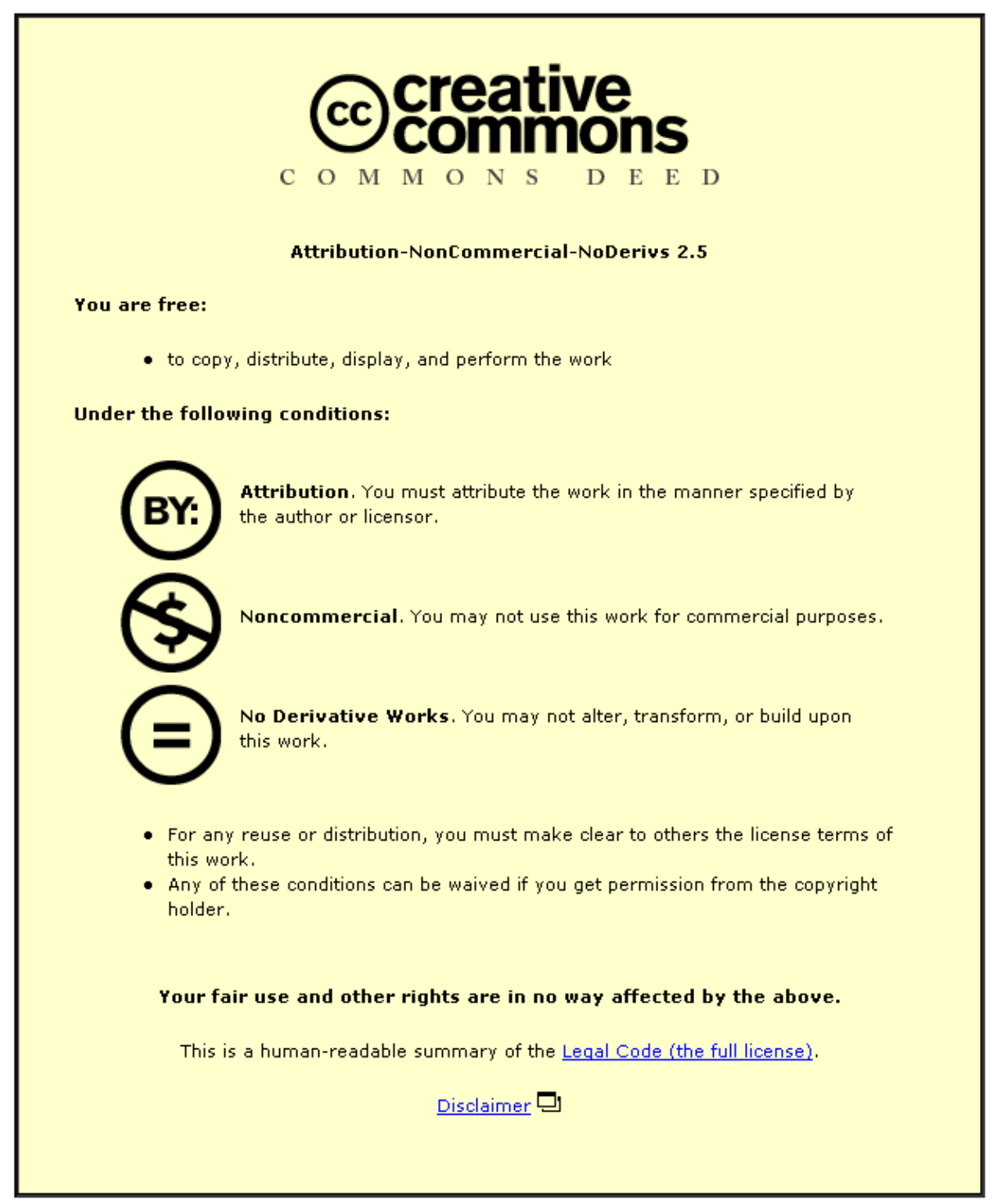

For the full text of this licence, please go to: http://creativecommons.org/licenses/by-nc-nd/2.5/ 


\title{
A MIXED-COST BLIND ADAPTIVE RECEIVER FOR DS-CDMA
}

\author{
Peerapol Yuvapoositanon and Jonathon A. Chambers \\ Signal and Image Processing Group, \\ Department of and Electronic and Electrical Engineering, \\ University of Bath, \\ Bath BA2 7AY, United Kingdom \\ Email: eeppay@bath.ac.uk
}

\begin{abstract}
A new mixed-cost receiver for direct-sequence code-division multiple access (DS-CDMA) systems is proposed. An adaptive mixing function is introduced to combine the constrained minimum output energy (CMOE) and constant modulus (CM) criteria together. Simulations confirm the near-far resistance of the proposed receiver over a wide range of near-far situations.
\end{abstract}

\section{INTRODUCTION}

The constrained minimum output energy (CMOE) criterion $[1,2]$ is widely known as an effective interference cancellation scheme for code-division multiple access (CDMA) systems. This feature is emphasised when the channel exhibits a near-far environment: the situation when one or more interfering users have greater power than the desired user. The performance of the CMOE receiver degrades, however, in high signal to noise ratio (SNR) situations and by distortion of the received signals due to multipath fading channels [1]. In [2], the constraint proposed in [1] is replaced by a code constraint matrix to retain the output energy of the desired user at a particular path delay. Although this new scheme prevents the cancellation of the desired signal and sidesteps the use of an explicit constraint on the orthogonal vector [1], the performance of the CMOE receiver still degrades either in the case of low interference power or when the number of multipaths is extended [3].

The constant modulus algorithm (CMA) receiver performs better in (inverse) channel estimation and provides near-Weiner receiver performance [4]. However, since its cost surface is multimodal, the CM criterion possibly possesses some undesirable local minima which in some cases associate to the solutions of interfering users. Good initialisation for a CMA receiver can help evade these local minima and accelerates the convergence of the algorithm. In se-

This work was supported by the Mahanakorn University of Technology, Bangkok, Thailand. vere near-far environments, a pre-whitening process of the received signal is indispensable despite its excessive computational complexity [5].

This paper concerns the exploitation of the salient features of both criteria to produce a near-far resistant receiver which can be operated in multipath fading channels with a wide-range of near-far levels. The proposed algorithm jointly updates the receiver weight vector by adaptively minimising a mixed-cost function. The mixing parameter is also adapted according to the near-far level. Simulation results are provided to show the signal to interference plus noise ratio (SINR) performance of the proposed combining scheme compared to those of the existing algorithms. It is shown that the mixed-cost scheme is superior in terms of SINR levels over a wide-range of near-far levels in multipath fading channels.

\section{SYSTEM MODEL}

For the real system model, the baseband received signal for a $K$-user asynchronous CDMA channel is defined as

$$
r(t)=\sum_{i=-\infty}^{\infty} \sum_{k=1}^{K} A_{k} b_{k}[i] c_{k}\left(t-i T-\tau_{k}\right)+v(t)
$$

where $A_{k}$ represents the received amplitude of the $k$ th user. The data bits $b_{k}[i]$ are independent identically distributed (i.i.d.) and assumed to be drawn from the finite alphabet $\{-1,+1\}$. The symbol period is denoted by $T$. The spreading (or signature) waveform of the $k$ th user $c_{k}(t)$ is $L_{c^{-}}$ dimensional and has unit energy property, i.e., $\left\|c_{k}\right\|^{2}=1$ and $\tau_{k} \in[0, T)$ are the relative offsets of the asynchronous signals at the receiver. The zero-mean additive white Gaussian channel noise $v(t)$ has constant power spectral density $\sigma^{2}$. If we incorporate the amplitude $A_{k}$ and delay $\tau_{k}$ in the channel response $h_{k}(t)$, we can replace the spreading code sequence $c(t)$ with the discrete-time combined channel-code 
response

$$
g_{k}[l]=\sum_{i=0}^{L_{c}-1} c_{k}[i] h_{k}[l-i]
$$

where $c_{k}[i]$ is the $i$ th element of the code vector for the $k$ th user $\mathbf{c}_{k}=\left(c_{k}[0], \ldots, c\left[L_{c}-1\right]\right)^{T}$.

The continuous-time received signal $r(t)$ is sampled to form a length- $L_{f}$ received signal vector at the $n$th observation, where $L_{f}$ is the length of a receiver for the $k$ th user with tap-weight vector $\mathbf{f}_{k}$,

$$
\mathbf{r}[n]=\left(r\left[n N+L_{f}-1\right], \cdots, r[n N]\right)^{T} .
$$

The received signal vector $r(t)$ in (1) can then be formulated in the matrix-vector form as

$$
\mathbf{r}[n]=\sum_{k=1}^{K} \mathbf{r}_{k}[n]+\mathbf{v}[n]=\sum_{k=1}^{K} \mathbf{G}_{k} \mathbf{b}_{k}[n]+\mathbf{v}[n]
$$

where $\mathbf{G}_{k}$ is the combined code-channel response matrix of the $k$ th user and $\mathbf{b}_{k}[n]=\left(b_{k}\left[n+L_{b}-1\right], \cdots, b_{k}[n]\right)^{T}$ with $L_{b}=\left\lceil\frac{L_{f}+L_{h}-1}{L_{c}}\right\rceil$ and $\left.\mathbf{v}[n]=\left(v[n N]+L_{f}-1\right], \cdots, v[n N]\right)^{T}$. Note that

$$
\mathbf{G}_{k}=\mathbf{C}_{k} \mathbf{H}_{k},
$$

where the $L_{f} \times L_{b} L_{h}$ code matrix $\mathbf{C}_{k}$ and the $L_{b} L_{h} \times L_{b}$ channel matrix $\mathbf{H}_{k}$ are defined as

$$
\mathbf{C}_{k}=\left(\begin{array}{ccc}
c_{k}\left[L_{c}-1\right] & & \mathbf{0} \\
\vdots & \ddots & \\
c_{k}[0] & & c_{k}\left[L_{c}-1\right] \\
& \ddots & \vdots \\
\mathbf{0} & & c_{k}[0]
\end{array}\right), \mathbf{H}_{k}=\left(\begin{array}{llll}
\mathbf{h}_{k} & & & \\
& \mathbf{h}_{k} & & \mathbf{0} \\
\mathbf{0} & & \ddots & \\
& & & \mathbf{h}_{k}
\end{array}\right),
$$

where the channel response vector for the $k$ th user has length $L_{h}$, i.e., $\mathbf{h}_{k}=\left(h\left[L_{h}-1\right], \ldots, h[0]\right)^{T}$. For brevity, we shall consider the first user as the desired user and drop the subscript $k$ in all variables involving the first user.

\section{MIXED-COST ALGORITHM}

Consider a combined cost function

$$
\text { where } \begin{aligned}
J(\mathbf{f}, \lambda) & =\lambda \tilde{J}(\mathbf{f})+(1-\lambda) \tilde{\tilde{J}}(\mathbf{f}), \\
\tilde{J}(\mathbf{f}) & =E\left\{\mathbf{f}^{T} \mathbf{R} \mathbf{f}\right\} \\
\tilde{\tilde{J}}(\mathbf{f}) & =E\left\{\left(\left(\mathbf{f}^{T} \mathbf{r}\right)^{2}-1\right)^{2}\right\}
\end{aligned}
$$

are the CMOE [2] and the CM [6] cost functions respectively and $\lambda \in[0,1]$ is the mixing parameter and $\mathbf{R}=$ $E\left\{\mathbf{r r}^{T}\right\}$. The CMOE criterion [2] is given by

$$
\min _{\mathbf{f}} E\left\{\mathbf{f}^{T} \mathbf{R f}\right\} \quad \text { subject to } \mathbf{f}^{T} \mathbf{C}=\mathbf{1},
$$

where $\mathbf{1} \triangleq(1,0, \cdots, 0)^{T}$ since the first path is assumed to be the dominant path and the gradient of the CMOE cost is given by [2]

$$
\left.\frac{\partial \tilde{J}(\mathbf{f})}{\partial \mathbf{f}}\right|_{\mathbf{f}=\mathbf{f}[n]}=z[n] \boldsymbol{\Pi}_{\mathbf{C}}^{\perp} \mathbf{r}[n] .
$$

where $z[n]=\mathbf{f}^{T}[n] \mathbf{r}[n]$ is the output of the receiver and $\boldsymbol{\Pi}_{\mathbf{C}}^{\perp}=\mathbf{I}-\mathbf{C}\left(\mathbf{C}^{T} \mathbf{C}\right)^{-1} \mathbf{C}^{T}$ denotes the projection matrix onto the nullspace of $\mathbf{C}$. The CMA receivers, i.e., the locations in receiver parameter space of the local minima of $\tilde{\tilde{J}}(\mathbf{f})$, are found by means of the CMA algorithm [6] which searches adaptively for the zero of the gradient

$$
\left.\frac{\partial \tilde{\tilde{J}}(\mathbf{f})}{\partial \mathbf{f}}\right|_{\mathbf{f}=\mathbf{f}[n]}=z[n]\left(z^{2}[n]-1\right) \mathbf{r}[n] .
$$

For the derivation of a CMA receiver, two important points need to be mentioned. First, it should be noted that the initialisation of a CMA receiver is crucial for the convergence to the solution of a desired user. In [5], a timing acquisition scheme of a desired user is proposed in order to be used as an initialisation of a CMA equaliser. Collectively, this acquisition-equalisation process is called the minimumentropy CMA (ME-CMA) receiver [5]. When the received signals are not pre-whitened in the equalisation process, the ME-CMA receiver is essentially a conventional CMA receiver.

Second, the CMA receiver is shown to converge faster if a constraint is imposed on the received signals as shown in [7]. However, it can be shown that the CMA receiver still converges to a desired solution without the requirement of any constraint as long as an appropriate initialisation is used [5]. In the derivation of the mixed-cost receiver, therefore, we do not impose any constraint upon the constant modulus derivative.

\subsection{Weight vector update equation}

Following the derivation of the algorithms for both criteria, the update equation of the mixed cost CMOE-CMA receiver weight vector $\mathbf{f}[n]$ is given by

$$
\begin{aligned}
\mathbf{f}[n+1]= & \mathbf{f}[n]-\left.\mu \frac{\partial J(\mathbf{f}, \lambda)}{\partial \mathbf{f}}\right|_{\mathbf{f}=\mathbf{f}[n]} \\
= & \mathbf{f}[n]-\mu\left(\lambda z[n] \Pi_{\mathbf{C}}^{\perp} \mathbf{r}[n]\right. \\
& \left.\quad-\beta(1-\lambda) z[n]\left(z^{2}[n]-1\right) \mathbf{r}[n]\right),
\end{aligned}
$$

where $\mu$ is the stepsize of the mixed-cost algorithm. For best operation of this algorithm, it is necessary to weight the constant modulus derivative which in effect modifies the mixture in (6) and can be explained by the nonhomogeneity of the two costs. This is realised by introducing the scaling 
factor $\beta$ for the constant modulus derivative in (9). Note that for the case of $\lambda=1$, equation (9) is the update equation of the CMOE receiver [2] and when $\lambda=0$, the mixed-cost receiver is essentially the CMA receiver [6].

\subsection{Update equations for the mixing parameter}

The main objective of the derivation of the mixed-cost algorithm is to jointly exploit the features of the two criteria in various near-far environments. Therefore, the mixing parameter $\lambda$ is replaced by the time-varying version $\lambda[n]$ in order to track the variation of the channel.

We adopt the multi-step method as described in [8] for the update of the mixing parameter $\lambda[n]$ in a similar manner as for adapting the gain in the adaptive gain algorithm. The adaptation of the mixing parameter $\lambda[n]$ is obtained by applying a second LMS-type algorithm to adaptively minimise $J(\mathbf{f}, \lambda)$ with respect to $\lambda$. The stochastic gradient update equation for $\lambda[n]$ is given by

$$
\begin{aligned}
\lambda[n+1]= & {\left[\lambda[n]-\left.\alpha \frac{\partial J(\mathbf{f}, \lambda)}{\partial \lambda}\right|_{\lambda=\lambda[n]}\right]_{\lambda_{-}}^{\lambda_{+}} } \\
= & {\left[\lambda[n]-\alpha\left(z[n]^{2}-\left(z[n]^{2}-1\right)^{2}\right.\right.} \\
& +\left\{2 \lambda[n] z[n] \mathbf{r}^{T}[n]\right. \\
& \left.\left.\left.+4(1-\lambda[n])\left(z[n]^{2}-1\right) z[n] \mathbf{r}^{T}[n]\right\} \Psi[n]\right)\right]_{\lambda_{-}}^{\lambda_{+}}
\end{aligned}
$$

where $\alpha$ is the adaptation rate and $[\cdot]_{\lambda_{+}}^{\lambda_{+}}$denotes truncation to the limits of the range $\left[\lambda_{-}, \lambda_{+}\right]$and $0 \leq \lambda_{-}<\lambda_{+} . \Psi[n]$ represents the derivative $\partial \mathbf{f}[n] /\left.\partial \lambda\right|_{\lambda=\lambda[n]}$. From (9), the update equation of $\Psi[n]$ is given by

$$
\begin{aligned}
\Psi[n+1]= & {\left[I-\mu\left(\lambda[n] \mathbf{\Pi}_{\mathbf{C}}^{\perp} \mathbf{r}[n] \mathbf{r}[n]^{T}\right.\right.} \\
& \left.\left.-\beta(1-\lambda[n])\left(3 z^{2}[n]-1\right) \mathbf{r}[n] \mathbf{r}[n]^{T}\right)\right] \Psi[n] \\
& -\mu\left(z[n] \mathbf{\Pi}_{\mathbf{C}}^{\perp} \mathbf{r}[n]-\beta\left(z^{2}[n]-1\right) z[n] \mathbf{r}[n]\right) .
\end{aligned}
$$

Equation (9) together with (10) and (11) constitute the new mixed-cost CMOE-CMA algorithm. The structure of the proposed receiver is shown in Fig. 1.

\subsection{Computational complexity and convergence prop- erties}

The computational complexity of the algorithm is $L_{f}^{2}+$ $16 L_{f}+12$ and $L_{f}^{2}+9 L_{f}$ in terms of multiplications and additions. Since global convergence property of the CMOE has been given in [2] and local convergence of CMA has been shown in [4], with careful choice of $\mu$ and $\lambda[n]$, the combined algorithm should demonstrate at least similar convergence properties.

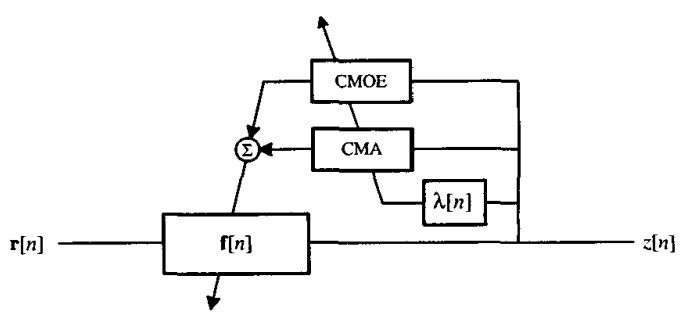

Fig. 1. The mixed-cost CMOE-CMA receiver.

\section{SIMULATIONS}

We considered a symbol-asynchronous system with processing gain $L_{c}=31$ and number of users $K=7$. User delays $\tau_{k}$ were uniformly distributed over $\left[0,7 T_{c}\right)$ and then kept fixed. The propagation channels are bandlimited with rootraised-cosine pulse shaping with excess bandwidth 0.2 . The number of multipath rays was three, where the last two rays were uniformly distributed in delay over $\left[0,7 T_{c}\right)$. The channel length for all users was $10 T_{c}$. We assumed without loss of generality that the first user is the user of interest with unity power. The timing of the first user was assumed to be known. The background noise was zero-mean AWGN with $\mathrm{SNR}=20 \mathrm{~dB}$ (referenced to the first user). Each receiver was length-2 $L_{c}$. The initial value of $\lambda[n]$ was set to unity and $\lambda_{-}$and $\lambda_{+}$were 0 and 1 respectively. The adaptation rate $\alpha$ was $5 \times 10^{-4}$ and $\Psi[0]$ was $0.1[1, \cdots, 1]^{T}$. The performance measure was the averaged SINR in $\mathrm{dB}$ and all SINR plots were averaged over 100 Monte-Carlo runs. We compared the performances of the CMA receiver, the CMOE receiver [2] and the proposed mixed-cost $\mathrm{CMOE}$ CMA receiver. We tested the performances of the three receivers in various settings of the near-far situations which can be quantified in terms of the near-far ratio (NFR) where $\mathrm{NFR}=10 \log _{10} \frac{A_{k}^{2}}{A_{1}^{2}}, \forall k=\{2, \ldots, 7\}$. Fig. 2 (a) and (b) show the averaged SINR plots of the three receivers at NFR= $14 \mathrm{~dB}$ and $26 \mathrm{~dB}$ respectively. It is observed that the performance of the CMA receiver is degraded in high NFR cases because the attraction basin of the desired user is likely to reduce in dimension as the NFR increases. The CMOE receiver reveals the characteristic of near-far resistance as shown in Fig. 2 (b) but inferior to the CMA receiver in the low NFR cases as in Fig. 2 (a). In both cases, the mixed-cost receiver is superior to the two existing receivers. The steady-state averaged SINR plots at various NFRs are shown in Fig. 3. For the mixed-cost receiver, high SINR levels were maintained over a wide-range of near-far levels confirming its near-far resistance characteristic. Time evolution plots of $\lambda[n]$ for different NFRs are shown in Fig. 4. Notice that the relaxation rate is varied as a function of the NFR settings. For low NFRs, $\lambda[n]$ decays quickly to zero 


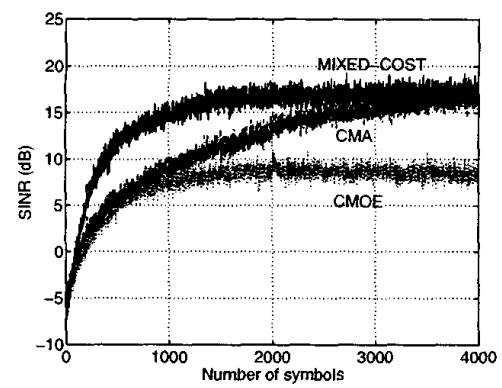

(a)

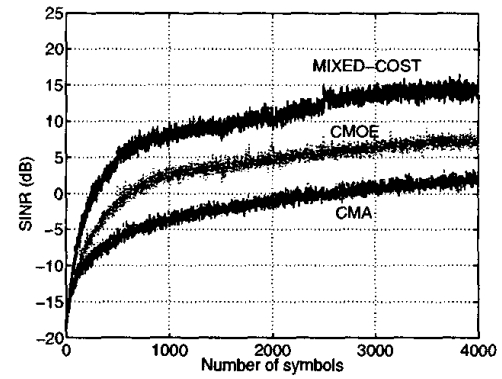

(b)

Fig. 2. The comparison of SINR performances of three receivers at (a) $\mathrm{NFR}=14 \mathrm{~dB}$ and (b) $\mathrm{NFR}=26 \mathrm{~dB}$.

whereas its magnitude is sustained at high levels for high NFRs.

\section{CONCLUSION}

We have presented a new mixed-cost receiver structure for DS-CDMA systems based on the CMOE and CM criteria. The multi-step method is exploited in the derivation of the adaptive mixing parameter algorithm. Simulations have confirmed that the averaged SINR performance of the proposed mixed-cost algorithm in various near-far situations is superior to the existing algorithms. On-going research is focused upon the evaluation of this method in the presence of time-varying interference.

\section{REFERENCES}

[1] M. L. Honig, U. Madhow, and S. Verdú, "Blind adaptive multiuser detection," IEEE. Trans. Inform. Theory, vol. 41, pp. 944-966, 1995.

[2] M. K. Tsatsanis, "Inverse filtering criteria for CDMA systems," IEEE Trans. Signal Processing, vol. 45, no. 1, pp. 102-112, 1997.

[3] P. Schniter and C. R. Johnson Jr., "On the robustness of

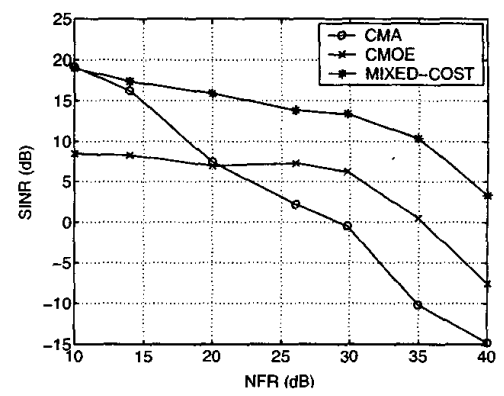

Fig. 3. The plots of SINR in $\mathrm{dB}$ as NFR varies from $10 \mathrm{~dB}$ to $40 \mathrm{~dB}$.

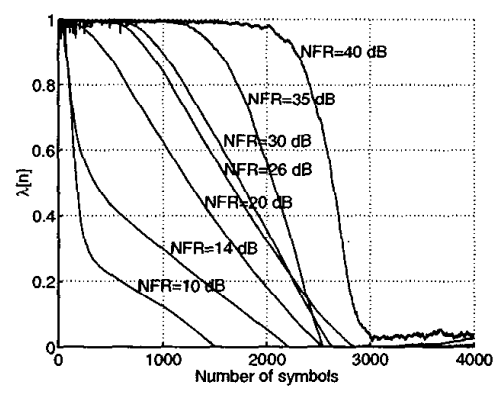

Fig. 4. The plots of $\lambda[n]$ as NFR varies from $10 \mathrm{~dB}$ to 40 dB.

blind linear receivers for short-code CDMA," in Proc. IEEE 2nd SPAWC, Annapolis, MD, pp. 13-16, 1999.

[4] H. H. Zeng, L. Tong, and C. R. Johnson Jr., "Relationships between the constant modulus and Wiener receivers," IEEE Trans. Inform. Theory, vol. 44, no. 4, pp. 1523-1538, 1998.

[5] P. Schniter and C. R. Johnson Jr., "Minimum-entropy blind acquisition/equalization for uplink DS-CDMA," in Proc. Allerton Conf. on Commun., Contr., and Computing, Monticello, IL, pp. 401-410, 1998.

[6] D. N. Godard, "Self-recovering equalization and carrier tracking in two-dimensional data communication systems," IEEE Trans. Commun., vol. 28, pp. 1867-1875, 1980.

[7] L. Li and H. Howard Fan, "Blind CDMA detection and equalization using linearly constraint," in Proc. Int. Conf. Acoustics, Speech and Signal Processing, Istanbul, Turkey, vol. 5, pp. 2905-2908, 2000.

[8] A. Benveniste, M. Métivier, and P. Priouret, Adaptive Algorithms and Stochastic Approximations, Volume 22 of Applications of Mathematic, Springer-Verlag, New York, 1990. 